\title{
FAST INTERPOLATION FOR GLOBAL POSITIONING SYSTEM (GPS) SATELLITE ORBITS
}

\author{
Beny Neta \\ Naval Postgraduate School \\ Department of Mathematics \\ Code $\mathrm{MA} / \mathrm{Nd}$ \\ Monterey, CA 93943 \\ D. A. Danielson \\ Naval Postgraduate School \\ Department of Mathematics \\ Code MA/Dd \\ Monterey, CA 93943
}

\author{
C. P. Sagovac \\ 819 South Charles Street \\ Baltimore, MD 21230-3938
}

J. R. Clynch

Naval Postgraduate School

Department of Oceanography

Code $\mathrm{OC} / \mathrm{Cl}$

Monterey, CA 93943

June 2, 1996

\begin{abstract}
In this report, we discuss and compare several methods for polynomial interpolation of Global Positioning System ephemeris data. We show that the use of difference tables is more efficient than the method currently in use to construct and evaluate the Lagrange polynomials.
\end{abstract}

\section{$\underline{\text { Introduction }}$}

The problem of interpolating Global Positioning System (GPS) precise, post fit ephemeris data is an important aspect of geodetic work utilizing GPS. Given that a high accuracy $(<1 \mathrm{~m})$, high precision $(1 \mathrm{~cm})$ orbit can be generated though the use of dense observations and special integrations, it is necessary to interpolate these ephemeris at high accuracy to utilize these orbits.

These high accuracy orbits are produced by several organizations (DMA, NGS, JPL, several Universities) and are widely available. An ephemeris typically consists of satellite positions at evenly spaced times over a week. Most ephemeris are given at $900 \mathrm{sec}$ (15 min) time steps although the NGS ones are at $1200 \mathrm{sec}(20$ min). The GPS satellites are in $12 \mathrm{hr}$ circular orbits making 900 sec ephemeris steps $7.5 \mathrm{deg}$ of arc.
The typical geodetic user collects GPS data at intervals from $1 \mathrm{sec}$ to $30 \mathrm{sec}$ and needs to find the satellite position at the times of that data. The times needed are really not the evenly spaced received times, but the transmit times that are about $60 \mathrm{msec}$ before reception. A precise value for this propagation delay is not known until the solution process is partially done. Therefore usually one needs to find a cluster of satellite positions a few msec from a nominal evenly spaced interval.

In the past the typical technique used by the DMA [Malys, 1989], NGS [Remondi, 1991], JPL [Watkins, 1995] and others is a Lagrange interpolation. The orders vary from $8^{t h}$ to $11^{t h}$. This approach directly computes the value of the function (the three Cartesian Earth centered earth fixed coordinates) from the unique polynomial going through the data points. The coefficients are not found, and finding them may introduce errors [Press et al, 1992]. Several evaluations of the accuracy of this method [Remondi, 1991, Smith and Curtis, 1983] have been made. It is generally found that an $8^{\text {th }}$ order Lagrangian interpolation using $900 \mathrm{sec}$ data with the unknown in the center of the points gives values that compare with numerical integration at the $1 \mathrm{~cm}$ level.

The problem addressed here is to find if a more efficient numerical method that achieves the same accuracy can be used. This is motivated by the move- 


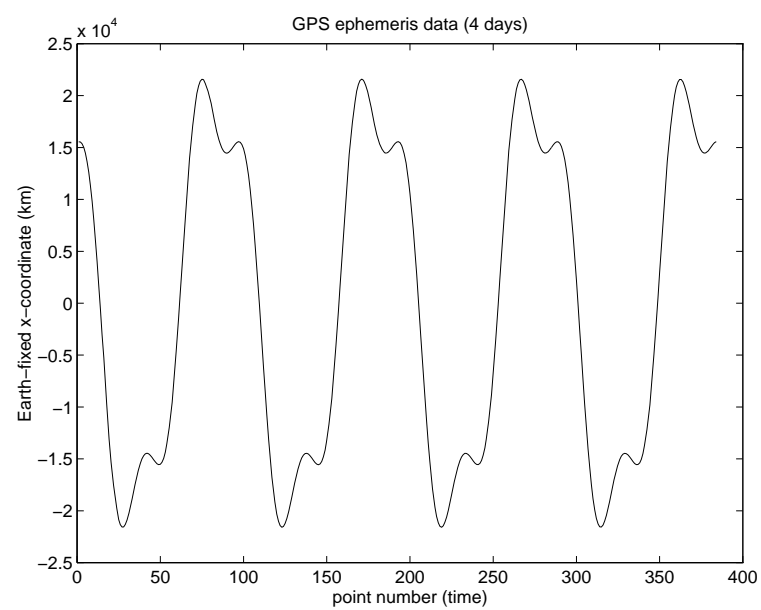

Figure 1: 4 day $x$-coordinate GPS ephemeris data at 900 second intervals

ment of processing from mainframes to PC's (486's and above).

Several aspects unique to the GPS satellites make this problem of interpolating the data different from the general problem of interpolation. Though we are interpolating GPS satellite orbital position data, it may be that the methods here are applicable to a broader class of problems. Where possible, we intend to take full advantage of the special geometry of the GPS satellite orbits.

A typical precise ephemeris orbit is supplied over an interval of eight days. Each ephemeris overlaps 1 day at each end with another ephemeris. It consists of position data which is Earth-centered, Earthfixed Cartesian coordinates given every 900 seconds $(t, x, y, z)$. We will not include velocity data which may in some cases be available. A plot of the data shows that it is "almost" periodic with a period of 24 hours or 96 intervals of 900 -second each. Of course the data would be periodic in inertial coordinates, however the data is given in a rotating frame (Earth centered - Earth fixed). This is done to place several subtle effects, such as polar motion, into the ephemeris generation problem. The user then does not have to have access to current data for geophysical effects to compute an Earth fixed position solution. Figure (1) is a plot of $x$-coordinate data (in kilometers) over a four day period. Note that the plot is with respect to the node (point) number which ranges from 1 to 384 . It is convenient to use node numbers since we may choose to map the interval of interest into different subintervals. For instance, the
Lagrange interpolation method maps the interval of interest into the interval $[-1,1)$ while the trigonometric polynomial interpolation method maps the interval of interest into the interval $[0,2 \pi)$. Later we will use the node numbers to compare the residuals for different methods.

There are two lengths of computation time in which we are interested for this problem. One time length is the generation time of the function (interpolant) which interpolates the evenly-spaced data. The other is the time needed to evaluate the interpolant at a point. For polynomial interpolation these might be the calculation of the coefficients of the polynomial interpolating the data and the time needed to evaluate that polynomial, once generated, at a particular point. As we shall see, however, there are clever ways in which to minimize the amount of work done in generating the desired data, and the two times might not be easily distinguished in these cases. In recommending a particular technique for interpolation it is important to know whether the interpolant will be evaluated once (or just a few times) on an interval or if it will be evaluated many times throughout the interval. With a cluster of times to be interpolated on an interval, the cost of generating the interpolant should be amortized over the set of times. This means that the time needed to generate the interpolant (a one-time cost) would not be as much a concern as the time needed to evaluate the interpolant at each of the desired times (a recurring expense). On the other hand, if one or a very few points were to be calculated on a given interval, the time needed to generate the interpolant would probably be more significant a concern than the time needed to evaluate it at the desired times since in general the generation of an interpolant is much costlier (in time) than its evaluation. This is a similar argument as one finds in deciding whether to use Gaussian elimination or LU factorization in solving systems of linear equations.

One method of polynomial interpolation involves performing the interpolation at a point without actually calculating the coefficients of the interpolating polynomial. This involves many less operations than the evaluation of a polynomial of degree $n$ at a particular point. This is a common technique currently employed. If we do not explicitly calculate the interpolant, however, we will in general still need to calculate some quantities prior to interpolation. For instance, we shall see that divided differences would need to be available prior to using the nested multiplication algorithm used in evaluating the Newton form of the interpolating polynomial.

Since ephemeris data is generated for an eight day 
time period, we have the opportunity to "front load" our work at the time of ephemeris receipt. By calculating needed data in advance we should be able to shorten the real time operation count. Thus we will be more concerned with the rapid evaluation of interpolants for specific times than their rapid generation. Of course in some cases the times of evaluation and generation will be closely related, as mentioned above. In others, they will be quite different and our hope will be to shift as much of the work as possible to the generation of efficient interpolants so as to allow the rapid evaluation of those interpolants.

In summary, we will describe the following methods:

- Lagrange polynomial interpolation

- Newton's divided difference interpolation polynomial

- Difference Tables

- Cubic Spline interpolation

- Trigonometric polynomial interpolation

- Tshebyshev polynomial interpolation

We will describe the advantages and disadvantages of each of these methods for the problem of interest, namely for the efficient interpolation at clusters of points. The actual codes used to prepare the Figures and Table in this report are available on the Internet at URL address http://math.nps.navy.mil/ bneta.

\section{Lagrange Polynomial Interpolation}

Before we begin our investigation, it is necessary to describe the method which is currently being used. Simply put, given the $n+1$ ephemeris values $f\left(t_{0}\right), f\left(t_{1}\right), \ldots, f\left(t_{n}\right)$ at the distinct times $t_{0}, t_{1}, \ldots, t_{n}$, there exists a unique interpolating polynomial $p_{n}$ satisfying

$$
p_{n}\left(t_{i}\right)=f\left(t_{i}\right), \quad i=0,1, \ldots, n
$$

This polynomial can be written in the form (called the Lagrange interpolation polynomial)

$$
p_{n}(t)=\sum_{i=0}^{n} f\left(t_{i}\right) l_{i}(t)
$$

where

$$
\begin{aligned}
l_{i}(t)= & \frac{\left(t-t_{0}\right)\left(t-t_{1}\right) \cdots\left(t-t_{i-1}\right)}{\left(t_{i}-t_{0}\right)\left(t_{i}-t_{1}\right) \cdots\left(t_{i}-t_{i-1}\right)} \times \\
& \frac{\left(t-t_{i+1}\right) \cdots\left(t-t_{n}\right)}{\left(t_{i}-t_{i+1}\right) \cdots\left(t_{i}-t_{n}\right)}
\end{aligned}
$$

The eleventh order Lagrange method uses twelve data points to generate an eleventh order polynomial according to equation (1). This polynomial can then be evaluated at desired times within the interval of interest. The error $R_{n}(t)$ in using the Lagrange interpolant $p_{n}(t)$ to estimate the function $f(t)$ (having at least $n+1$ derivatives throughout the open interval) at some point $t$ can be written [Buchanan and Turner, 1992]:

$$
R_{n}(t)=f(t)-p_{n}(t)=\frac{L_{n+1}(t) f^{(n+1)}(\xi)}{(n+1) !}
$$

where $\xi$ is some point in the interval $\left[t_{0}, t_{n}\right]$ and

$$
L_{n+1}(t)=\prod_{i=0}^{n}\left(t-t_{i}\right)
$$

One difficulty in implementing high degree polynomial interpolation routines of any kind is the fact that the error between the interpolating polynomial and the data or function being interpolated grows rapidly near the endpoints of the interval over which the interpolation is being performed. For this reason the eleventh order Lagrange method is overlapped as successive intervals are chosen within the ephemeris (we call this walk-along interpolation). Due to the high accuracy requirements, only the center subinterval is interpolated for each Lagrange polynomial which is generated. Whereas the initial interval spans points one through twelve, the second interval spans points two through thirteen in order to provide the highest degree of accuracy. The first eleventh order Lagrange polynomial would then be used for times between points six and seven, while the second polynomial would be valid for times between points seven and eight. The numerical accuracy of this method has been verified to the $1 \mathrm{~cm}$ level for the data we are interpolating [Remondi, 1991].

Difficulties arise in that the process of creating and evaluating the resulting eleventh order polynomials is computationally expensive. The cost of evaluating the Lagrange form at a point is provided by de Boor [1978]. It is

$$
(2 n-2) A+(n-2) M+(n-1) D
$$


for each of the $n+1$ numbers $l_{i}(t)$, where $A$ denotes an addition or subtraction and $M$ and $D$ denote multiplication and division, respectively. Forming equation (1) then takes another

$$
(n-1) A+n M
$$

operations, leaving the total count per component of the position vector at

$$
(3 n-3) A+(2 n-2) M+(n-1) D
$$

This is the number of operations per component in the implementation of Lagrange interpolation. A simple modification of the algorithm would reduce the amount of work to

$$
(2 n-1) A+n M+n D
$$

operations (see de Boor [1978]). It consists of first forming the quantities

$$
Y_{i}=\frac{f\left(t_{i}\right)}{\prod_{j \neq i}\left(t_{i}-t_{j}\right)}, \quad i=1, \ldots, n
$$

Afterwards, $p_{n}(t)$ is calculated through

$$
\begin{gathered}
\phi_{n}(t)=\prod_{i=1}^{n}\left(t-t_{i}\right) \\
p_{n}(t)=\phi_{n}(t) \sum_{i=1}^{n} Y_{i} /\left(t-t_{i}\right)
\end{gathered}
$$

This method is somewhat faster than using (1)-(2) and is easy to implement. In addition, no loss of accuracy occurs in its implementation. If the time $t$ is very close to one of the interpolating points $t_{i}$, one must be careful in computing $p_{n}(t)$ because of the division of $Y_{i}$ by a very small number.

\section{Newton's Divided Difference Interpolation Polynomial}

A more efficient means by which we may form the interpolating polynomial is through divided differences. We follow the developments given in Buchanan and Turner [1992] and de Boor [1978]. Suppose we have $n+1$ distinct interpolation points $t_{0}, t_{1}, \ldots, t_{n}$. Define the zeroth divided difference at $t_{i}$ by $f\left[t_{i}\right]=f\left(t_{i}\right)$. The first divided difference at $t_{i}, t_{j}$ is defined by

$$
a_{0}=f\left[t_{i}, t_{j}\right]=\frac{f\left[t_{i}\right]-f\left[t_{j}\right]}{t_{i}-t_{j}}
$$

and the $k$ th divided difference $f\left[t_{0}, t_{1}, \ldots, t_{k}\right]$ is defined by

$$
\begin{aligned}
a_{k} & =f\left[t_{0}, t_{1}, \ldots, t_{k}\right] \\
& =\frac{f\left[t_{0}, t_{1}, \ldots, t_{k-1}\right]-f\left[t_{1}, t_{2}, \ldots, t_{k}\right]}{t_{0}-t_{k}}, \quad k>0
\end{aligned}
$$

Newton's divided difference interpolation polynomial is the interpolation polynomial agreeing with the function $f$ at the points $t_{0}, t_{1}, \ldots, t_{n}$ and is given by

$$
\begin{aligned}
p_{n}(t) & =a_{0}+\left(t-t_{0}\right) a_{1}+\left(t-t_{0}\right)\left(t-t_{1}\right) a_{2}+\cdots \\
& +\left(t-t_{0}\right)\left(t-t_{1}\right) \cdots\left(t-t_{n-1}\right) a_{n}
\end{aligned}
$$

or, rearranging,

$$
\begin{aligned}
p_{n}(t) & =a_{0}+\left(t-t_{0}\right)\left\{a_{1}+\left(t-t_{1}\right)\left\{a_{2}+\cdots\right.\right. \\
& +\left(t-t_{n-2}\right)\{a_{n-1}+\left(t-t_{n-1}\right) a_{n} \overbrace{\} \ldots\}}^{n-1 \text { times }}
\end{aligned}
$$

This form consists of two additions and a multiplication per level in the expression. Since there are $n$ levels, the operation count is seen to be

$$
n(2 A+M)
$$

which is more economical than the standard Lagrange form. This leads us to the so-called nested multiplication or Horner's algorithm:

Given the $n+1$ distinct points $t_{0}, t_{1}, \ldots, t_{n}$ with associated coefficients $a_{0}, a_{1}, \ldots, a_{n}$, the value of the interpolating polynomial $p_{n}(t)$ for some $t \in\left[t_{0}, t_{n}\right]$ is given by $b_{0}$ according to the following iteration:

Set $b_{n}=a_{n}$

For $k=n-1$ to 0 by -1

$b_{k}=a_{k}+\left(t-t_{k}\right) b_{k+1}$

End For

By the uniqueness of the interpolating polynomial there can be no difference in comparison to Lagrange, but the gain in speed may be of importance for our purposes. Note that the divided differences $a_{k}$ can be calculated and stored in advance of the actual interpolation so that the operation counts given here reflect the operations needed at interpolation time. A total operation count would have to include the operations needed to generate the divided differences. Also, calls from storage may need to be counted, depending on system architecture considerations.

The divided difference algorithm does not take advantage of the fact that our interval sizes are fixed - we required the nodes to be distinct but made no restriction on the spacing between nodes. In the next section we will investigate the special case when the interval sizes are constant. 


\section{$\underline{\text { Difference Tables }}$}

The case of equally-spaced data points is a special case of Newton's divided differences and leads to other interpolation formulas. The error and operation counts for the methods presented here are essentially identical to those presented above. The formulas are given in their simplest form and should not be used for computation. A nested multiplication approach similar to the one described in the previous section should be used for each of these in order to minimize the cost of computation. One important aspect of this method is the determination of the differences and the method to be used to interpolate at a given time $t$. It will be necessary to include some code to determine which differences are to be used, though the differences themselves can be calculated when the given ephemeris becomes available. In addition, the chosen method will depend on the location of the interpolation time relative to the data times. Here we follow the description given in Buchanan and Turner [1992]. Our data occurs at times which can be expressed as

$$
t_{k}=t_{0}+k h
$$

where $t_{0}$ is a reference time for the interval of interest and $h$ is the constant steplength. We normally think of $k$ as being a positive integer and $t_{0}$ as being the initial time in the interval of interest but in this case we will only require $k$ to be an integer and $t_{0}$ to be any time corresponding to a data point in the interval. The sign of $k$ will depend on the reference time $t_{0}$ in relation to the time of interest. There are now several differences which can be defined, one of which is the forward difference.

The general forward difference $\Delta f\left(t_{i}\right)$ is given by

$$
\Delta f\left(t_{i}\right)=f\left(t_{i+1}\right)-f\left(t_{i}\right)=f\left(t_{i}+h\right)-f\left(t_{i}\right)
$$

Its powers are calculated recursively according to the following

$$
\begin{aligned}
\Delta^{k} f\left(t_{i}\right) & =\Delta\left(\Delta^{k-1} f\left(t_{i}\right)\right) \\
& =\Delta^{k-1} f\left(t_{i+1}\right)-\Delta^{k-1} f\left(t_{i}\right) \\
\Delta^{k} f_{i} & =\sum_{j=0}^{k}(-1)^{k-j}\left(\begin{array}{c}
k \\
j
\end{array}\right) f_{i+j}
\end{aligned}
$$

where we have introduced the notation $f_{i}=f\left(t_{i}\right)$. Additionally, the differences are related to divided differences by

$$
\Delta^{k} f\left(t_{i}\right)=k ! h^{k} f\left[t_{i}, t_{i+1}, \ldots t_{i+k}\right]
$$

An application of this last formula to equation (5) immediately yields a forward difference formula (called Newton's forward difference formula or the NewtonGregory forward difference formula). Here we assume that the degree of the interpolating polynomial is $n$ while the number of data points in our table is $N$ :

$$
\begin{aligned}
p_{n}(t) & =f_{0}+\frac{\left(t-t_{0}\right)}{h} \Delta f_{0}+\frac{\left(t-t_{0}\right)\left(t-t_{1}\right)}{2 h^{2}} \Delta^{2} f_{0}+\cdots \\
& +\frac{\left(t-t_{0}\right)\left(t-t_{1}\right) \cdots\left(t-t_{n-1}\right)}{n ! h^{n}} \Delta^{n} f_{0}
\end{aligned}
$$

A simple change of variable $\tau=\left(t-t_{0}\right) / h$ yields the compact form

$$
p_{n}(\tau)=\sum_{j=0}^{n}\left(\begin{array}{l}
\tau \\
j
\end{array}\right) \Delta^{j} f_{0}
$$

with the generalized binomial coefficients

$$
\left(\begin{array}{l}
\tau \\
j
\end{array}\right)=\frac{\tau(\tau-1) \cdots(\tau-j+1)}{j !}
$$

We measured actual run times of the methods (3) and (6) to construct the interpolating polynomial using 96 points, and then the time to evaluate (4) and (7) (using nested multiplication) for 9 and 12 points around the one requested. The results given in the table indicate that the use of difference tables may be slightly faster, if many evaluations are required.

\begin{tabular}{||l|cc|cc||}
\hline Method & \multicolumn{2}{|c|}{ Lagrange } & \multicolumn{2}{c||}{ Diff. Table } \\
\hline Order & $8^{t h}$ & $11^{t h}$ & $8^{t h}$ & $11^{t h}$ \\
\hline Construct & .0241 & .0242 & .0270 & .0280 \\
\hline Evaluate & .0309 & .0410 & .0308 & .0308 \\
\hline
\end{tabular}

Table 1: Run times in seconds

\section{Cubic Spline Interpolation}

Cubic spline interpolation is computationally efficient and has an advantage with respect to walk-along Lagrange because it allows the user to calculate the interpolating polynomials over the entire interval at one time, at the beginning of the interpolation process. This calculation involves solving a tridiagonal system of equations. Additionally, the fact that each subinterval is represented by a cubic polynomial means that evaluations on those intervals are much quicker 
than their eleventh order polynomial counterparts, making the cubic spline method a good choice where accuracy is less important than speed.

Here we will sketch the derivation of the cubic spline interpolation; thorough treatments of cubic spline interpolation can by found in [Ahlberg, Nilson and Walsh, 1967], [Buchanan and Turner, 1992], [de Boor, 1978], and [Press, 1992]. Given the $n+1$ ephemeris values $f\left(t_{0}\right), f\left(t_{1}\right), \ldots, f\left(t_{n}\right)$ at the distinct times $a=t_{0}, t_{1}, \ldots, t_{n}=b$, we construct a piecewise cubic interpolant $p$ to $f$ as follows. On each subinterval $\left[t_{i}, t_{i+1}\right]$ we wish to construct a cubic polynomial $p_{i}$ in such a way that the resulting interpolation formula over the entire interval is continuous in its first and second derivatives. The result is

$$
\begin{aligned}
p_{i}(t) & =A_{i}(t) f\left(t_{i}\right)+B_{i}(t) f\left(t_{i+1}\right)+C_{i}(t) f^{\prime \prime}\left(t_{i}\right) \\
& +D_{i}(t) f^{\prime \prime}\left(t_{i+1}\right), \quad i=0, \ldots, n-1
\end{aligned}
$$

where

$$
\begin{gathered}
A_{i}=\frac{t_{i+1}-t}{t_{i+1}-t_{i}}, \\
B_{i}=\frac{t-t_{i}}{t_{i+1}-t_{i}} \\
C_{i}=\frac{1}{6}\left(A_{i}^{3}-A_{i}\right)\left(t_{i+1}-t_{i}\right)^{2}, \\
D_{i}=\frac{1}{6}\left(B_{i}^{3}-B_{i}\right)\left(t_{i+1}-t_{i}\right)^{2}
\end{gathered}
$$

We do not yet have the $n+1$ values of $f^{\prime \prime}\left(t_{i}\right)$ needed for the determination of the solution, but application of the continuity of the first derivative on the entire interval leads us to the following equations for $i=$ $1,2, \ldots, n-1$ :

$$
\begin{aligned}
& \frac{t_{i}-t_{i-1}}{6} f^{\prime \prime}\left(t_{i-1}\right)+\frac{t_{i+1}-t_{i-1}}{3} f^{\prime \prime}\left(t_{i}\right) \\
+ & \frac{t_{i+1}-t_{i}}{6} f^{\prime \prime}\left(t_{i+1}\right) \\
= & \frac{f\left(t_{i+1}\right)-f\left(t_{i}\right)}{t_{i+1}-t_{i}}-\frac{f\left(t_{i}\right)-f\left(t_{i-1}\right)}{t_{i}-t_{i-1}} .
\end{aligned}
$$

Note that there are $n-1$ equations for $n+1$ unknowns, leaving two second derivatives undetermined. The choice of the two boundary conditions $f^{\prime \prime}(a)$ and $f^{\prime \prime}(b)$ provides the required unique solution. In our case it makes sense to use the periodicity, i.e. apply (8) and (9) for $i=n$ and enforce $p_{0}(t) \equiv p_{n}\left(t+t_{n}\right) \quad$ for $t_{0} \leq t \leq t_{1}$. As a consequence, $f\left(t_{0}\right)=f\left(t_{n}\right), \quad f\left(t_{1}\right)=f\left(t_{n+1}\right), \quad f^{\prime \prime}\left(t_{0}\right)=$ $f^{\prime \prime}\left(t_{n}\right)$, and $f^{\prime \prime}\left(t_{1}\right)=f^{\prime \prime}\left(t_{n+1}\right)$. Therefore if we use equal step size $h$, the cubic spline version of equation (9) can be written as

$$
\left(\begin{array}{cccccc}
4 & 1 & 0 & 0 & \cdots & 1 \\
1 & 4 & 1 & 0 & \ldots & 0 \\
0 & 1 & 4 & 1 & 0 & \cdots \\
\vdots & \vdots & \ddots & \ddots & \ddots & \\
0 & \cdots & 0 & 1 & 4 & 1 \\
1 & \cdots & 0 & 0 & 1 & 4
\end{array}\right)\left(\begin{array}{c}
f^{\prime \prime}\left(t_{1}\right) \\
f^{\prime \prime}\left(t_{2}\right) \\
f^{\prime \prime}\left(t_{3}\right) \\
\vdots \\
f^{\prime \prime}\left(t_{n-1}\right) \\
f^{\prime \prime}\left(t_{n}\right)
\end{array}\right)=\left(\begin{array}{c}
R_{1} \\
R_{2} \\
R_{3} \\
\vdots \\
R_{n-1} \\
R_{n}
\end{array}\right)
$$

where $R_{i}=24 f\left[t_{i-1}, t_{i}, t_{i+1}\right]$.

If accuracy can be sacrificed for speed then the cubic spline method may be preferred over any of the methods here. However, the $O\left(h^{4}\right)$ accuracy provided by the cubic spline may be insufficient for GPS satellite interpolation requirements.

\section{Trigonometric Polynomial Interpolation}

The roots of this method of interpolation can be traced to the beginning of the nineteenth century. Briggs and Henson [1995] present a brief history of this method, in particular the fact that Gauss used it around 1800 to interpolate the orbit of the asteroid Pallas. The preceding methods are standard interpolation techniques typically used for continuous, differentiable functions defined on compact intervals. No other special information about the functions being interpolated is exploited by these methods. It is at this point that we examine some special properties of our GPS ephemeris data. As previously mentioned, our ephemeris data is supplied over an interval of eight days and consists of Earth-centered, Earthfixed Cartesian coordinate position data given every 900 seconds. A plot of the data in Figure (1) shows that it is very close to periodic, and it is for this reason that we examine the trigonometric polynomial interpolation method.

Due to the fact that the position data has a period of twenty-four hours, we restrict our attention to a single twenty-four hour period and generate a trigonometric polynomial $\rho_{n}$ using all the data available over that period. Again, we must remember that our satellite orbit is not truly periodic, but very close to that in inertial coordinates. Since the error incurred by assuming the data to be periodic over a $j$ day period would be almost $j$ times as great as the error incurred from assuming the orbit to be periodic over a single day, we discourage use of this routine over intervals exceeding the fundamental period of the data. In order to minimize the effects of the assumption of periodicity one should interpolate over a single period. 
The idea in generating our interpolant is to assume that the data is from a periodic function of time defined over the interval $[0,2 \pi)$ which can be represented by a trigonometric polynomial of the form

$$
\rho_{n}(t)=a_{0}+\sum_{k=1}^{n}\left(a_{k} \cos k t+b_{k} \sin k t\right)
$$

In our problem we simply map the twenty-four hour interval of interest into the interval $[0,2 \pi)$ using a linear change of variable. In deriving the coefficients we follow the discussion of interpolating polynomials and the Fast Fourier Transform found in Buchanan and Turner [1992]. Clearly, the above equation can be written as

$$
\rho_{n}(t)=\sum_{k=-n}^{n} \gamma_{k} e^{i k t}
$$

where the coeffcients are given by

$$
\begin{gathered}
\gamma_{k}=a_{k}+i b_{k} \quad k=-n, \ldots,-1 \\
\gamma_{k}=a_{k}-i b_{k} \quad k=1, \ldots, n
\end{gathered}
$$

and, of course, $\gamma_{0}=a_{0}$. If we denote the point on the unit circle corresponding to $t \in[0,2 \pi)$ by

$$
\xi=e^{i t}
$$

we may then write

$$
\rho_{n}(t)=\sum_{k=-n}^{n} \gamma_{k} \xi^{k}
$$

If we denote the points corresponding to nodes by

$$
\xi_{j}=e^{i t_{j}}=\cos t_{j}+i \sin t_{j}
$$

the interpolation problem is to find the coefficients satisfying

$$
\rho_{n}\left(t_{j}\right)=\sum_{k=-n}^{n} \gamma_{k} \xi_{j}^{k}=f\left(t_{j}\right)
$$

where $f\left(t_{j}\right)$ are the function values for $j=$ $0,1, \ldots, 2 n$. For an odd number of nodes, it can be shown that

$$
\gamma_{p}=\frac{1}{2 n+1} \sum_{k=0}^{2 n} f_{k} \xi_{k}^{-p} \quad p=-n,-n+1, \ldots, n
$$

Unfortunately, as pointed out by Buchanan and Turner [1992], the operation count for this discrete Fourier Transform is $O\left(n^{2}\right)$ making it too expensive

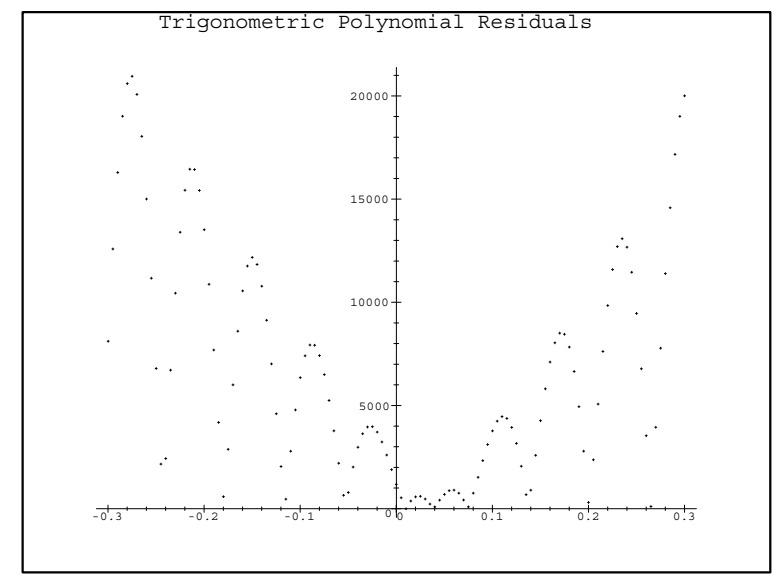

Figure 2: Trigonometric polynomial residuals $(\mathrm{cm})$

for practical application. Luckily, there is a faster way to calculate the discrete Fourier coefficients.

Suppose that we have the case where the number of nodes is of the form $n=2^{N}$. The task is then to find the trigonometric polynomial $\rho_{n}$ which interpolates $f$ at the uniformly spaced data set $t_{0}, t_{1}, \ldots, t_{n-1}$. In this case the trigonometric polynomial can be calculated by using the Fast Fourier Transform, which has an operation count of $O(n \log n)$. This makes it an attractive method for our purposes and we will refer to it as the FFT method. Unfortunately, however, over a twenty-four hour period our data will consist of ninety-six points separated by intervals of 900 seconds. Though ninety-six is not a power of two, we nevertheless can use $n=2^{5}=32$ points in the interval to test our trigonometric polynomials. We simply select every third point in the interval and calculate the trigonometric polynomial which interpolates at those thirty-two points. We may then compare the Lagrange method to the FFT method by choosing some subinterval having a length of 36 nodes, twelve of which are used to generate the eleventh order Lagrange interpolating polynomial. These twelve nodes (equally spaced with 3 times the spacing) should coincide with twelve of the thirty-two nodes used in the FFT method.

The above calculations were performed with the aid of Maple and a plot of residuals in centimeters is shown in Figures (2) and (3). A linear change of variable was used to map the trigonometric polynomial defined on the interval $[0,2 \pi]$ into the interval $[-1,1]$. Since we have taken every third point, the last one is at $93 \pi / 48$ rather that $2 \pi$. This is the reason why the figures do not show symmetry about 0 which is $\pi$ in the original domain. 


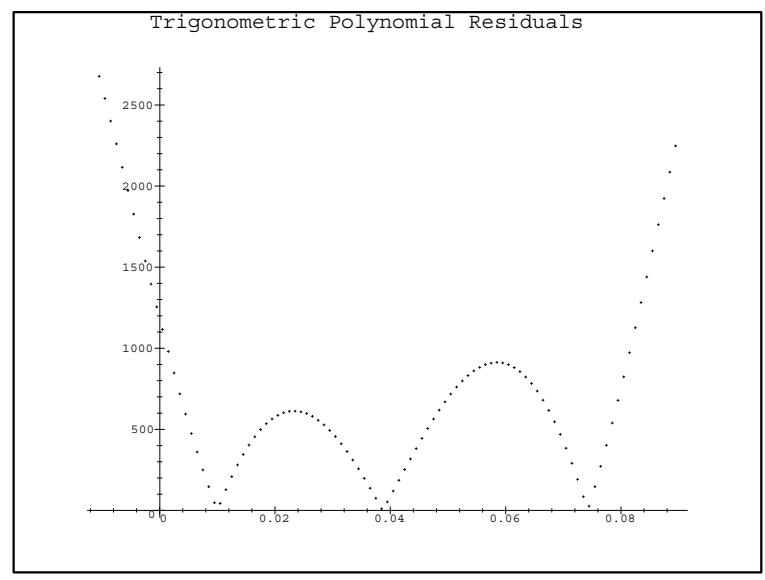

Figure 3: Trigonometric polynomial residuals $(\mathrm{cm})$, (zoomed)

The subinterval chosen for this analysis was located at the center of the set of nodes in order to show the location where the trigonometric interpolant is most accurate. Examination of Figure (3) reveals that on a single subinterval the trigonometric polynomial method agrees with the Lagrange interpolation method to within only ten meters.

The effects of error growth near the ends of the intervals for the FFT method could be handled by shifting the twenty-four hour period over which the trigonometric polynomial is derived, placing the data point squarely in the center for the most accurate work. A bound on the mean square error incurred by approximating the periodic function $f$ from which the data is sampled by the interpolant $\rho$ is given in Briggs and Henson [1995] as

$$
\|f-\rho\| \leq \frac{C}{N^{p+1}}
$$

where $N$ is the number of data points, $C$ is a constant and the periodic extension of $f$ has $(p-1)$ continuous derivatives, $p \geq 1$. Since continuity of the periodic extension of $f$ is required for this bound we cannot use it unless the function is truly periodic on the chosen interval. Briggs and Henson [1995] perform a trigonometric interpolation on an arbitrary polynomial defined on $[-1,1]$ and comment that it is not unreasonable to suspect the mean square errors to decrease as $N^{-1}$.

Brigham (1988) presents the Cooley-Tukey algorithm for the case when $N$ is not necessarily a power of 2. Using this idea, we can use all 96 points which will cut the error by a factor of 3 .

Another concern will be the time required to evaluate the interpolant, so it is of interest to discuss the rapid calculation of the trigonometric polynomials, in particular the terms

$$
\cos \pi t, \sin \pi t, \cos 2 \pi t, \sin 2 \pi t, \ldots, \cos n \pi t, \sin n \pi t .
$$

Using the trigonometric summation formulas one can write the well-known recursion [Goertzel, 1960]

$$
\begin{gathered}
\sigma_{k}=\cos k \pi t \\
\tau_{k}=\sin k \pi t \\
\left(\begin{array}{c}
\sigma_{k+1} \\
\tau_{k+1}
\end{array}\right)=\left(\begin{array}{cc}
\sigma_{1} & -\tau_{1} \\
\tau_{1} & \sigma_{1}
\end{array}\right)\left(\begin{array}{c}
\sigma_{k} \\
\tau_{k}
\end{array}\right) \quad k=1,2, \ldots
\end{gathered}
$$

which requires only two trigonometric calculations in order to recover all the needed terms. Of course, the growth of round-off errors should always be checked when using a long sequence (i.e. $n$ large).

It is important that we use all the available data in forming the trigonometric polynomial $\rho_{n}(t)$ because the mean square error involved in using the FFT method depends so critically on the number of data points. Since the DFT seems to be computationally expensive, we must resort to another algorithm.

\section{Tshebyshev Polynomial Interpolation}

In order for us to understand Tshebyshev polynomial interpolation, it is necessary to re-examine the polynomial interpolation of $n+1$ data points on a given interval. Given $n+1$ points on an interval, the $n$th degree polynomial which interpolates those points over the given interval is unique. Of course this does not prevent us from writing the polynomial in a number of different ways.

Before we describe what Tshebyshev polynomial interpolation is, it may be a good idea to say what it is not. Tshebyshev polynomial interpolation is not the expression in the Tshebyshev polynomial basis of an $n$th order polynomial which interpolates $n+1$ arbitrary data points on a given interval. We are free to choose any suitable polynomial basis for expressing such a polynomial, but we must remember that the results of any calculations performed using that polynomial will be the same regardless of how the polynomial is expressed; simply writing a given polynomial in a different basis does not alter it. Therefore the residuals which are produced when using these different forms of the same polynomial will be the same.

However, another set of $n+1$ points on the same interval would yield a different polynomial. As it turns out, polynomial interpolation is sensitive to the distribution of the points being interpolated. If we were 
allowed to choose the points on an interval to be interpolated, we would find that certain choices of $n+1$ points would yield polynomials which did a better job of interpolating than others. As stated, our data points are evenly spaced throughout the interval of interest, so that will not have the luxury of choosing a preferred set of $n+1$ points on any interval unless we are able to generate more data. The following discussion closely follows the treatment given by Press et al [1992]. The Tshebyshev polynomial of degree $n$ on $[-1,1]$ is defined as

$$
T_{n}(t)=\cos (n \arccos t)
$$

It follows that the Tshebyshev polynomials satisfy the three term recurrence relation

$$
T_{n+1}(t)=2 t T_{n}(t)-T_{n-1}(t) \quad n=1,2, \ldots
$$

In addition,

$$
\int_{-1}^{1} \frac{T_{n}(t) T_{k}(t)}{\sqrt{1-t^{2}}}=0, \quad k \neq n
$$

and

$$
\int_{-1}^{1} \frac{T_{n}(t) T_{k}(t)}{\sqrt{1-t^{2}}}= \begin{cases}\pi & k=n=0 \\ \pi / 2 & k=n \neq 0\end{cases}
$$

so that the Tshebyshev polynomials are orthogonal on the interval $[-1,1]$ with respect to the weight function $w(t)=1 / \sqrt{1-t^{2}}$. The first few Tshebyshev polynomials are given by

$$
\begin{gathered}
T_{0}(t)=1 \\
T_{1}(t)=t \\
T_{2}(t)=2 t^{2}-1 \\
T_{3}(t)=4 t^{3}-3 t \\
T_{4}(t)=8 t^{4}-8 t^{2}+1 \\
T_{5}(t)=16 t^{5}-20 t^{3}+5 t
\end{gathered}
$$

It can be shown that the zeros of $T_{n}(t)$ on $[-1,1]$ are given by

$$
t_{j}=\cos \left[\frac{\pi(j-1 / 2)}{n}\right] \quad j=1,2, \ldots, n
$$

and that the Tshebyshev polynomials satisfy a discrete orthogonality condition [Press et al, 1992]

$$
\sum_{j=1}^{n} T_{i}\left(t_{j}\right) T_{k}\left(t_{j}\right)= \begin{cases}0 & i \neq k \\ n / 2 & i=k \neq 0 \\ n & i=k=0\end{cases}
$$

The powers of $t$ can also be expressed in the Tshebyshev polynomial basis.

Any function $f(t)$ can be approximated by a finite linear combination of Tshebyshev polynomials, i.e.

$$
f(t)=\frac{1}{2} a_{0}+\sum_{i=1}^{N} a_{i} T_{i}(t)
$$

where

$$
a_{i}=\frac{2}{\pi} \int_{-1}^{1} \frac{f(t) T_{i}(t)}{\sqrt{1-t^{2}}} d t .
$$

These coefficients $a_{i}$ can be computed numerically using the $M$ equally spaced data points

$$
\begin{gathered}
t_{j}=-1+j \Delta t, \quad j=0,1, \cdots, M-1 \\
\Delta t=\frac{2}{M-1}
\end{gathered}
$$

e.g. via the composite midpoint rule (we have to avoid evaluating the integrand at the endpoints, $t_{0}$ and $\left.t_{M-1}\right)$

$$
a_{i}=\frac{2}{\pi} \sum_{j=0}^{M-2} \frac{\frac{1}{2} f\left(t_{j+1 / 2}\right) T_{i}\left(t_{j+1 / 2}\right)}{\sqrt{1-t_{j+1 / 2}^{2}}} \Delta t,
$$

where $f\left(t_{j+1 / 2}\right)$ can be approximated by a quadratic or cubic polynomial. For example to use cubic splines to approximate the integrand, we get

$$
\frac{2}{\pi}\left[\frac{h^{4}}{4} \sum_{j=0}^{M-2} a_{j}+\frac{h^{3}}{3} \sum_{j=0}^{M-2} b_{j}+\frac{h^{2}}{2} \sum_{j=0}^{M-2} c_{j}+h \sum_{j=0}^{M-2} d_{j}\right]
$$

where the integrand $g(t)$ is approximated on the interval $\left[t_{j}, t_{j+1}\right]$ by the cubic polynomial

$$
g(t)=a_{j}\left(t-t_{j}\right)^{3}+b_{j}\left(t-t_{j}\right)^{2}+c_{j}\left(t-t_{j}\right)+d_{j}
$$

We can also use the midpoint rule on the two leftmost panels and the two rightmost panels only. On the rest of the panels we can use the fourth order Simpson's $\frac{1}{3}$ rule. This will yield the following approximation for the coefficients:

$$
\begin{aligned}
a_{i}= & \frac{\Delta t}{\pi}\left\{4 g_{2}+\frac{2}{3} g_{3}+\frac{8}{3} g_{4}+\frac{4}{3} g_{5}+\right. \\
& \left.\cdots+\frac{8}{3} g_{M-3}+\frac{2}{3} g_{M-2}+4 g_{M-1}\right\},
\end{aligned}
$$

where

$$
g_{j}=\frac{f\left(t_{j}\right) T_{i}\left(t_{j}\right)}{\sqrt{1-t_{j}^{2}}}
$$


If the number of panels left for Simpson's $\frac{1}{3}$ rule is not even, we can take the trapezoidal rule over one of the panels (in here we took the third from last panel). For example, we show below the formula for an odd number of panels:

$$
\begin{aligned}
a_{i}= & \frac{\Delta t}{\pi}\left\{4 g_{2}+\frac{2}{3} g_{3}+\frac{8}{3} g_{4}+\frac{4}{3} g_{5}+\right. \\
& \left.\cdots+\frac{8}{3} g_{M-4}+\frac{5}{3} g_{M-3}+g_{M-2}+4 g_{M-1}\right\} .
\end{aligned}
$$

To evaluate $f(\tau)$ we then use the following recursion:

$$
\begin{gathered}
d_{N+1}=d_{N+2}=0 \\
d_{j}=2 \tau d_{j+1}-d_{j+2}+a_{j}, \quad j=N, N-1, \cdots, 2,1 \\
f(\tau)=d_{0}=\tau d_{1}-d_{2}+\frac{1}{2} a_{0} .
\end{gathered}
$$

The benefit of Tshebyshev expansion is that the maximum deviation on the interval is minimized. Thus it will not suffer from the disadvantage of higher order polynomials, that is the error here doesn't grow rapidly near the endpoints of the interval. Therefore we eliminate the need for "walking" interpolator.

\section{Conclusions}

This has been a preliminary study of various interpolation methods for GPS ephemeris data. The alternate long-arc methods of interpolation we have tried so far yield much greater than $1 \mathrm{~cm}$ error when compared to the short-arc eleventh order Lagrange polynomial. However, more work should be done before completely ruling out these alternate methods.

Our study does indicate that the use of difference tables should be more efficient than the direct method currently used to construct and evaluate the Lagrange polynomials.

\section{Acknowledgement}

This research was supported in part by the Naval Postgraduate School Research Program. The authors gratefully acknowledge the partial support of NISE West Coast Division.
Ahlberg, J. H., Nilson, E. N., and Walsh, J. L., 1967, The theory of Splines and Their Applications, (New York: Springer Verlag)

Briggs, W.L. and Henson, V. E., 1995, The DFT, An Owner's Manual for the Discrete Fourier Transform (Philadelphia: SIAM)

Brigham, E. O., 1988, The Fast Fourier Transform and its Applications, (New Jersey: Prentice Hall)

Buchanan, J.L. and Turner, P.R., 1992, Numerical Methods and Analysis, (New York: McGraw-Hill, Inc.)

de Boor, C., 1978, A Practical Guide to Splines, vol. 27 of Applied Mathematical Sciences, (New York: Springer-Verlag)

Goertzel, G., 1960, Fourier Series, Mathematical Methods for Digital Computers, (New York: Wiley)

Malys, S., and Ortiz, M. J., 1989, Geodetic absolute positioning with differenced GPS carrier beat phase data, Proceedings of the $5^{\text {th }}$ International Symposium on Satellite Positioning, 13-17 March 1989, Las Cruces, NM.

Press, W. H., Flannery, B. P., Teukolsky, S. A., and Vetterling, W. T., 1992, Numerical Recipes in FORTRAN, The Art of Scientific Computing, 2nd Edition, (New York: Cambridge University Press)

Remondi, B. W., 1989, Extending the National Geodetic Survey Standard GPS Orbit Formats, NOAA Technical Report NOS 133 NGS 46, (Rockville, MD: US Department of Commerce, NOAA)

Remondi, B. W., 1991, NGS Second Generation ASCII and Binary Orbit Formats and Associated Interpolation Studies, (Rockville, MD: US Department of Commerce, NOAA)

Smith, R. and Curtis, V., 1983, Interpolation Study, Naval Surface Weapons Center Dahlgren Laboratory Memorandum.

Watkins, M., 1995, Private communication.

\section{References}

